\title{
Análisis comparado de los Grados y Dobles Grados de Pedagogía y Educación Social en la universidad española. Profesionales para la equidad social en el siglo XXI.
}

\author{
Cáceres-Muñoz,Jorge \\ Universidad de Extremadura, Cáceres,España \\ jorgecm@unex.es \\ Rosado-Castellano,Fátima \\ Universidad de Extremadura, Cáceres,España \\ marosadoc@alumnos.unex.es \\ Martín-Sánchez,Miguel \\ Universidad de Extremadura, Cáceres,España \\ miguelmartin@unex.es
}

\section{Resumen}

La Pedagogía y la Educación Social son dos disciplinas propias de las Ciencias de la Educación con una naturaleza dual -teórica y práctica-, que les hace ser -desde una visión pestalozziana-, cabeza, corazón y mano en el mundo educativo y social de hoy. Se ha asistido en Europa y más notablemente en España, al deterioro y desmantelamiento del Estado de bienestar. Esta situación requiere de un urgente rearme y empoderamiento de la sensibilidad social por parte de la ciudadanía. Sin embargo, el entendimiento de la sociedad actual necesita de esa sensibilización y/o motivación para con los problemas y metas sociales. Para llegar a ello se necesitan profesionales de la educación con alta diversidad competencial. Desde este contexto, este trabajo utiliza el método comparado propio de la Educación Comparada con el objetivo de realizar un análisis de los Grados y Dobles Grados de Pedagogía y Educación Social en la universidad española. Se pretende reflexionar sobre el estado actual y el futuro de estos estudios clave para el empoderamiento social y la coherencia educativa. El número y titularidad de los centros que los imparten, sus planes de estudio, las salidas profesionales ofrecidas, las posibilidades de cooperación internacional o la conexión con el tejido institucional de las ciudades o barrios pretenden ser unidades intra-nacionales de comparación desde la mirada a las universidades españolas. Todo ello teniendo en cuenta el origen y evolución de estos estudios en España y las vicisitudes económicas actuales por las que pasa la institución universitaria. El ideal de sociedad se alcanzará entendiendo a esta formación como una inversión formativa protectora de la equidad social de las próximas décadas.

\section{Abstract}

Pedagogy and Social Education are two disciplines of the Educational Sciences with a dual nature - theoretical and practical - that makes them be -from a pestalozzian vision-, head, heart and hand in the educational and social world of today. We have witnessed in Europe and more notably in Spain, the deterioration and dismantling of the welfare state. This situation requires an urgent rearmament and empowerment of social sensitivity on the part of citizens. However, the understanding of the current Society does not only need that awareness and / or motivation for social problems and goals. To achieve this, education professionals with a high diversity of skills are needed. From this context, this work uses the compared method of Comparative Education with the objective of performing an analysis of the Degrees and Double Degrees of Pedagogy and Social Education in the Spanish University. The aim is to reflect on the current state and on the future of these key studies for social empowerment and educational coherence. The number and ownership of the centers that offer them, their study plans, the professional opportunities offered, the possibilities of international cooperation or the connection with the institutional fabric of the cities or neighborhoods are intended to be intra-national comparison units from the point of view of the Spanish universities. All this, taking into account the origin and evolution of these studies in Spain and the current economic vicissitudes through which the university institution passes. The ideal is achieved by understanding this training as a formative investment that protects social equity in the comingdecades.

Palabras clave: Pedagogía, Educación Social, Educación Comparada, Universidad, Equidad.

Keywords: Pedagogy, Social Education, Comparative Education, University, Equity. 


\section{INTRODUCCIÓN}

La Universidad española ha caminado en los últimos años hacia un proceso de convergencia que ha tenido sus puntos fuertes pero también su dosis de vulnerabilidad (De la Calle Velasco, 2004). Dentro de ese peaje quizá debemos detenernos en el alejamiento o falta de respuesta a las demandas sociales como hecho negativo que requiere una notable mejora. Los estudios que centran la mirada en el análisis de la Universidad (Senent Sánchez, 2005) pretender presentar datos sobre su estado y sobre su capacidad de mejoría y continuo perfeccionamiento. Esta es en definitiva la meta de este trabajo. Poner la mirada sobre los estudios de Pedagogía y Educación Social en España es acercarnos a conocer el capital humano que está saliendo de las universidades para dar respuestas a un amplio abanico de frentes abiertos en esta sociedad en crisis. Una sociedad que, no olvidemos, está saliendo de un duro proceso de recesión económica, donde el Estado de Bienestar se ha visto perjudicado y donde la desigualdad entre distintos grupos sociales se ha acrecentado. España experimentó desde 1978 un proceso de evolución en su calidad social, algo representado en la construcción y consolidación del Estado de Bienestar. Ello ha estado protagonizado por la aprobación de nuevos derechos sociales, el establecimiento de una política fiscal y una estructura de gasto público que elevó la calidad de vida de la ciudadanía en términos bastante extensos (Fernández García, 2012). Sin embargo, España ha vivido en los últimos diez años un episodio alarmante de crisis económica, política, social e institucional (Ramió, Matas, 2013), lo que ha afectado sobremanera a la calidad de los servicios públicos, las transferencias sociales, las intervenciones normativas, y las intervenciones públicas, dejando un escenario de desigualdad patente que es necesario reconstruir.

La irrupción de las Tecnologías de la Información y la Comunicación y su constante proceso de actualización, la globalización, el multiculturalismo, y los cambios en la estructura familiar, entre otros, se suman a la inestabilidad económica y política antes aludida. Todo ello ha creado oportunidades pero también incertidumbre, inseguridad y desigualad significativa, reforzándose dinámicas negativas ya naturalizadas por la amplia mayoría social como el consumismo, la superficialidad y el individualismo (Martínez García, 2018).

Desde este contexto, el objetivo de este trabajo ha sido realizar un análisis de los estudios de Pedagogía y Educación Social en España con el propósito de valorar la posibilidad de diseñar un doble grado que mejore la eficacia de la formación de ambos títulos. Para ello se ha optado por utilizar una metodología de carácter descriptivo en la que se pretende comparar la oferta de las distintas universidades en España. Para llevar a acabo este estudio comparado de aproximación se han obtenido datos de los planes de estudio, documentos de Practicum, movilidad y salidas profesionales disponibles en la web de 60 universidades españolas. La condición de las universidades que han sido consultadas ha sido tanto pública como privada y las titulaciones analizadas han sido las relativas a estudios de Grado en Pedagogía y Grado en Educación Social.

Las universidades y titulaciones consultadas han sido las siguientes: Universidad Abierta de Cataluña, Universidad Nacional de Educación a Distancia, Universidad complutense de Madrid, Universidad Politécnica de Valencia, Universidad de Barcelona, Universidad de Valencia, Universidad Internacional de La Rioja, Universidad Rey Juan Carlos I, Universidad de Jaén, Universidad de Granada, Universidad Autónoma de Barcelona, Universidad de Málaga, Universidad Autónoma de Madrid, Universidad de Oviedo, Universidad de Sevilla, Universidad Pompeu Fabra, Universidad Politécnica de Madrid, Universidad de Salamanca, Universidad de Alicante, Universidad de Zaragoza, Universidad de Santiago de Compostela, Universidad de Almería, Universidad Europea de Madrid, Universidad Carlos III de Madrid, Universidad de La Laguna, Universidad Católica de Murcia, Universidad Politécnica de Cataluña, Universidad de Extremadura, Universidad Internacional de Valencia, Universidad de Islas Baleares, Universidad Jaume I, Universidad de las Palmas de Gran Canaria, Universidad de Girona, Universidad Pública de Navarra, Universidad de la Rioja, Universidad Antonio de Nebrija, Universidad de Valladolid, Universidad de Murcia, Universidad Francisco de Vitoria, Universidad de León, Universidad de Cádiz, Universidad de Vigo, Universidad Ramón Llull, Universidad Isabel I, Universidad CEU San Pablo, Universidad a Distancia de Madrid, Universidad de Burgos, Universidad Camilo José Cela, Universidad Pontificia de Salamanca, Universidad Pablo de Olavide, Universidad Rovira y Virgili, Universidad del País Vasco, Universidad Católica de Valencia San Vicente Mártir, Universidad de Córdoba, Universidad de La Coruña, Universidad de Lleida, Universidad de Huelva, Universidad de Castilla La Mancha, Universidad Central de Cataluña y Universidad de Deusto.

Consultando las web institucionales de las universidades así como los planes de estudios de cada titulación se han tratado de valorar indicadores que tienen que ver con el tipo de universidad, es decir, si se trata de una universidad pública o privada, la nomenclatura de las asignaturas, el reparto de crédito, las 
posibilidades de movilidad y diversidad de los destinos de prácticas, etc. Con todo ello, se busca esclarecer las distintas modalidades existentes para la formación en Pedagogía y Educación Social en España, así como entrever una prospectiva prudente de hacia dónde pueden ir encaminadas estas titulaciones.

\section{ESTUDIO COMPARADO DE LAS TITULACIONES DE PEDAGOGÍA Y EDUCACIÓN SOCIAL EN LA UNIVERISDAD ESPAÑOLA}

\subsection{Número y titularidad de los centros}

El número total de centros analizados es de 60 , tomando finalmente de muestra 37 ya que son en los que se imparten alguna de las dos titulaciones estudiadas. En este caso encontramos 19 de Pedagogía y 35 de Educación Social, teniendo por tanto 17 centros coincidentes que imparten ambos grados. De estos centros señalar que 33 de ellos son públicos, ocupando un $89 \%$ del total de la muestra. Actualmente no se está impartiendo el doble grado de Pedagogía y Educación Social en ninguna universidad Española.

\subsection{Datos acerca de los planes de estudio}

En lo referente a los planes de estudio, la disposición de créditos es semejante en las distintas universidades y ambas titulaciones, siendo grados de 240 créditos, divididos en 60 créditos de formación básica en todas, teniendo diferencias menores entre el resto de asignaturas y las titulaciones. Esto es, en Pedagogía los créditos obligatorios oscilan entre 72 y 144, las optativas entre 18 y 60, el Trabajo Final de Grado entre 6 y 18 y las Practicas Externas entre 16 y 42 créditos. Referente a las Menciones, solo 4 de las universidades estudiadas ( $21 \%$ del total de universidades que imparten Pedagogía) tienen la opción de cursar Mención. En cuanto a Educación Social los créditos obligatorios oscilan entre 84 y 144, las optativas entre 6 y 60, el Trabajo Final de Grado entre 6 y 12 y las Prácticas Externas entre 18 y 43 créditos. Referente a las Menciones, solo 2 de las universidades estudiadas (6\% del total de universidades que imparten Educación Social) tienen la opción de cursar Mención.

Para el estudio pormenorizado de estas disciplinas, se ha tomado muestra de sus asignaturas, registrando en Pedagogía un total de 798 asignaturas (175 formación básica, 341 obligatorias y 282 optativas), asimismo cada una de ellas cuenta con Prácticas externas y TFG. Estas asignaturas han sido concentradas en un total de 339 grupos según su naturaleza. De ello se ha cuantificado la frecuencia con la que cada uno de estos grupos se repite (entre ambas titulaciones hay diferencias con respecto al \% de frecuencia dado el tamaño de las dos muestras). En el caso de Pedagogía se ha tomado una frecuencia $15.8 \%$, siendo éstos grupos de asignaturas las que se repiten 3 veces, obteniendo un total de 82 grupos de asignaturas: Política y legislación educativa; Teoría de la Educación; Pedagogía Social; Sociología de la Educación; Psicología del Desarrollo; Economía de la Educación; Educación Comparada; Historia de la Educación; Intervención educativa con alumnos con necesidades educativas especiales; Orientación Educativa; Didáctica General; Antropología de la Educación; Dirección, organización y gestión de centros educativos; Orientación profesional y laboral; Diagnóstico en Educación; Actividades de integración en distintas áreas; Evaluación de programas, centros y profesores; Formación en las organizaciones; Psicología de la Educación; Gestión y desarrollo de recursos humanos; Organización y gestión de instituciones educativas; Comunicación y Educación; Métodos de investigación e innovación educativa; Inglés; Diseño de programas de orientación; Educación para la cooperación y el desarrollo; Tecnología de la Información y la Comunicación en Educación; Tecnología Educativa; Diseño, desarrollo e innovación curricular; Filosofía de la Educación; Atención a la diversidad; Dificultades de aprendizaje; Diseño y evaluación de materiales didácticos; Procesos psicológicos básicos; Educación permanente; Educación a lo largo de la vida; Educación Intercultural; Educación para el empleo; Entornos virtuales de enseñanza-aprendizaje; Formación y desarrollo profesional del profesorado; Metodología de investigación en educación; Metodología de evaluación en educación; Pedagogía laboral; Historia de la Educación en España; Derecho, desarrollo y bienestar social; Estadística aplicada a la Educación; Pedagogía Diferencial; Diagnóstico pedagógico; Asesoramiento y apoyo educativo; Asesoramiento pedagógico; Educación para la igualdad; Formación, asesoramiento y desarrollo profesional; Formación en red y entornos virtuales de aprendizaje; Historia del pensamiento pedagógico; Intervención pedagógica con adolescentes y en situaciones de riesgo; Innovación educativa; Investigación educativa; Orientación escolar, familiar y social; Pedagogía familiar; Fundamentos de la sociedad contemporánea; Orientación educativa y acción tutorial; Técnicas de recogida y análisis de información en educación; Análisis de datos en educación; 
Aspectos evolutivos y educación del alumnado con deficiencias auditivas, motóricas y visuales; Desarrollo y ética profesional aplicada; Diagnóstico e intervención en sujetos con necesidades educativas especiales; Educación para la ciudadanía; Evaluación de centros y profesores; Evaluación de instituciones y organizaciones educativas; Evaluación de procesos de enseñanza; Formación laboral y ocupacional; Formación y actualización de la función pedagógica; Mediación Educativa; Legislación educativa y perspectiva internacional; Organización y dirección de centros educativos y formativos; Pedagogía ambiental; Planificación e intervención educativa; Psicobiología; Psicología Social; Recursos didácticos y tecnológicos en educación; Sociedad, ciencia y cultura; Historia de la Educación de las mujeres.
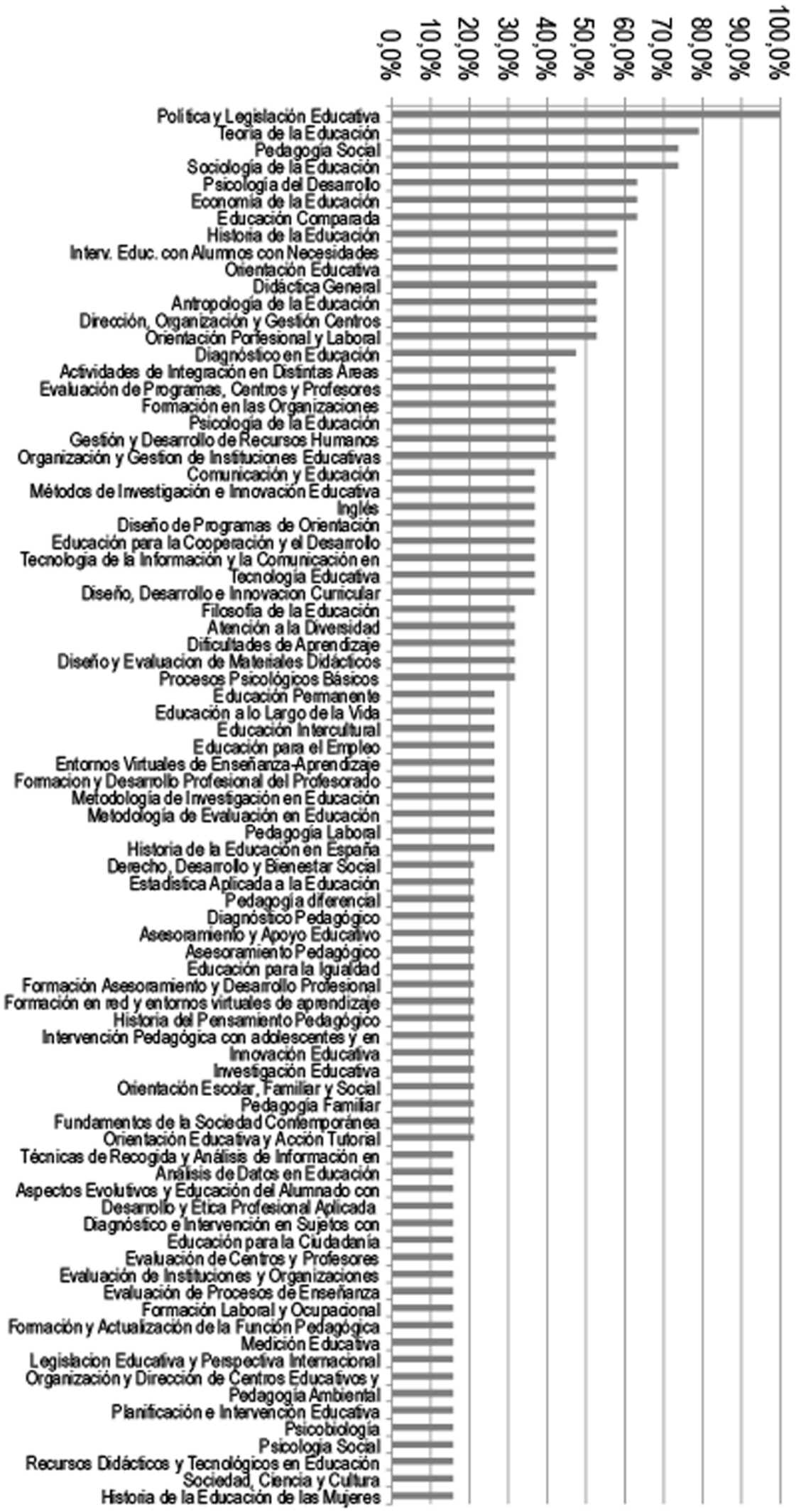

Figura 1. Frecuencia de aparición de grupo de asignaturas en Pedagogía: Elaboración Propia. 
En lo que respecta al Grado de Educación Social el total de asignaturas registradas es de 1453, entre las cuales se encuentran 336 formación básica, 614 obligatorias y 503 optativas, asimismo cuenta con Practicas externas y Trabajo Final de Grado. Estas asignaturas han sido concentradas en un total de 726 grupos según su naturaleza. La frecuencia de repetición es de $14.3 \%$, siendo éstos grupos de materias aquellos que se repiten 5 veces, obteniendo un total de 59 grupos de asignaturas: Pedagogía Social; Psicología del desarrollo humano; Psicología Social; Sociología de la Educación; Tecnología de la información y la comunicación en Educación; Animación sociocultural; Evaluación de programas e instituciones; Investigación socioeducativa; Mediación y Técnicas de resolución de conflictos; Metodología de investigación en ciencias sociales; Inglés; Educación con personas adultas; Política y legislación educativa; Teoría de la Educación; Antropología Social; Educación permanente; Historia de la Educación; Educación ambiental; Organización y gestión de instituciones socioeducativas; Diseño, desarrollo e innovación de planes, proyectos y programas socioeducativos; Intervenciónen drogodependencias; Orientación e inserción sociolaboral; Educación para la igualdad; Educación para la salud; Educación, cooperación y desarrollo sostenible; Intervención con personas con discapacidad; Métodos y técnicas de investigación; Acción socioeducativa con personas adultas; Artes plásticas y acción socioeducativa; Didáctica General; Política social y estado del bienestar; Servicios sociales; Acción socioeducativa en drogodependencias; Contextos y ámbitos de intervención en Educación Social; Desarrollo comunitario; Educación del ocio y el tiempo libre; Infancia y adolescencia en riesgo; Psicología de los grupos; Psicopatología; Antropología de la Educación; Música y acción social; Arte y cultura en Educación Social; Ética y deontología profesional; Diagnóstico de necesidades en Educación Social; Dinámica de grupos; Dirección y gestión de organizaciones socioeducativas; Diseño de programas y proyectos de Educación Social; Diversidad e igualdad en Educación; Economía; Educación para la ciudadanía; Educación Intercultural; Estadística aplicada a la educación; Habilidades sociales; Intervención educativa en problemas de desadaptación; Intervención en familias para la protección de la infancia; Intervención familiar; Pedagogía del ocio; Planificación de la intervención socioeducativa; Psicología de laEducación. 

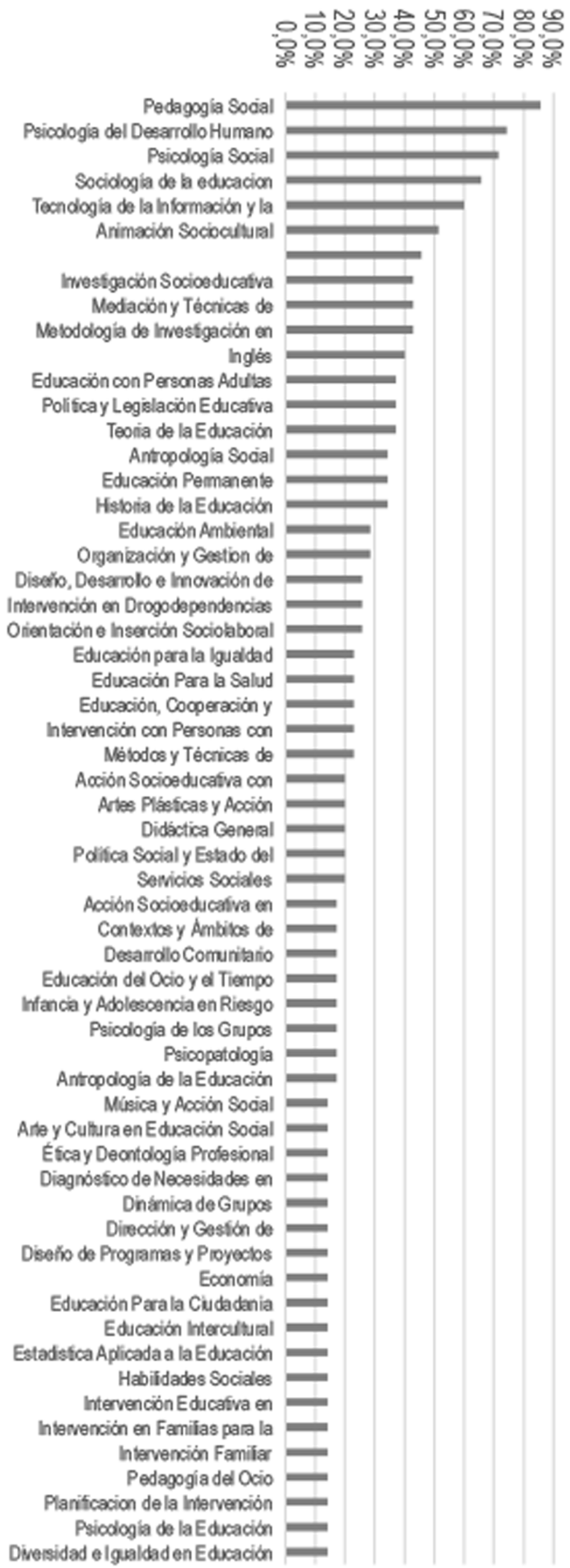

Figura 2. Frecuencia de aparición de grupo de asignaturas en Educación Social: Elaboración Propia. 


\section{DISCUSIÓN Y CONCLUSIONES}

De acuerdo con el escenario social al que aludíamos al inicio de este trabajo estamos obligados, como ya planteara Ortega Esteban (2005), a repensar la educación, a reflexionar sobre la práctica educativa y lo realizado en las instituciones para de alguna manera comprender el cúmulo de transformaciones que hemos ido enunciando y así establecer propuestas a la luz de una mejora para la equidad. En este sentido, los profesionales de la Pedagogía y la Educación Social tienen un difícil pero sobresaliente papel que cumplir. En este mundo interconectado pero insostenible, la gestión del capital, -que no solo es monetario sino también representado en bagaje de conocimiento y cultura-, debe ser también abordada por las instituciones de educación superior (Sánchez Alba et al, 2017). Son la plataforma de formación no solo de futuros ciudadanos y ciudadanas sino concretamente de aquellos profesionales destinados a actuar de salvavidas de la equidad.

La Educación irrumpe con mayúsculas en el escenario actual. Como alude Viñao (2009), el hecho educativo-formativo existe y es a su vez innegablemente social y de relevancia permanente. Esto plantea la incuestionable necesidad de que sea necesario estudiarlo y repensarlo desde perspectivas científicas, con la meta no solo de conocerlo sino también de transformarlo y mejorarlo. El pedagogo y el educador social, como profesionales del hecho educativo deben ser adalides de esta acción en pos de conseguir amortiguar las consecuencias de la crisis actual del Estado de Bienestar. La educación aparece como una herramienta que brinda al individuo posibilidades más certeras para su realización personal, que a la vez persigue su perfeccionamiento y la siempre necesaria inclusión de las personas y grupos en su contexto social. Ello viene a traducirse en un elemento vital para la prevención de los procesos de exclusión social gracias a la asimilación de un conjunto de competencias y valores propios y necesarios para cada entorno cultural (Castillo Sánchez y Gamboa Araya, 2012).

Buscar nuevas formas de mejorar la formación de los pedagogos y educadores sociales es contribuir no solo a la justificación de su existencia -tanto desde el ámbito científico, disciplinar y profesional-, sino también a mejorar su acción para con la sociedad. Ello se traduce en la obtención de frutos desde múltiples frentes y permite hacer frente a la distancia y desencuentros entre el campo académico y el profesional que numerosos autores han destacado (Úcar, 2011). Por ejemplo, para el entendimiento de la actual política educativa. Como señala Puelles (2007), la Educación lleva siendo durante décadas un terreno de batalla. La Educación se encuentra en manos del partidismo lo que evita el tan ansiado consenso. El plano ideológico se representa y se hace vivo en el vaivén político generando cambios constantes que pesan negativamente en el desarrollo educativo de un país, en este caso España. Por ello, formar profesionales competentes en el entendimiento de esta realidad, permitirá estar más cerca de una protección eficaz de los valores y fines básicos de la educación de las sociedades avanzadas y comprometidas no solo con el presente sino también con el futuro.

Otro ejemplo se representa en la posibilidad de estos profesionales de ejecutar proyectos o programas donde no solo intervienen para conocer la realidad social sino que son perfectos animadores de su conciencia, potenciando así el sentimiento de comunidad, tolerancia y altruismo propio de sociedades avanzadas. Ello hace real el objetivo de alcanzar la calidad de vida de los sujetos, mejorando la inclusión y con ello la participación activa de aquellos en situación de desventaja social (Cáride,2002).

Para ello desde la Pedagogía se debe seguir reforzando el saber pedagógico, saber complejo y frágil que plantea retos desde múltiples esferas; la disciplinariedad o saber acumulado de contendidos; la didáctica o el saber dedicado al arte de enseñar; la comprensión holística de las ciencias de la educación, lo que sirve para ubicarse en el conocimiento conceptual pero también en la práctica y en los valores asociados a esta; el saber investigar, para analizar la realidad, conocer sus límites y expandirlos para la mejora de la misma; y por último, el saber hacer, la praxis en sí misma, lo que sitúa en una forma correcta de ejecutar el saber y desarrollar el ser profesional (Houssaye, 2014).

Del mismo modo, desde la Educación Social se debe seguir llenando el vacío entre instituciones y reactivando una visión total del fenómeno educativo que va más allá de los espacios formales que, por ejemplo, un centro educativo de educación infantil y primaria representan. A día de hoy, todavía es prioritario seguir trabajando en lo que señalaran Puelles y Torreblanca (1995): la combinación y entendimiento de los espacios de educación formal y no formal. Sin olvidar la potencialidad que ofrece la educación informal, representada por ejemplo en los medios de comunicación (García Aretio, Ruiz Corbella y García Blanco, 2011). Hoy se sigue reivindicando la identidad del educador social como profesional de la educación cuya labor trasciende el sistema educativo reglado (Cáride, 2002). Una identidad basada en la operatividad (Pérez Serrano, 2005) como respuesta a los amplios espacios y permanentes tiempos en los que se puede y debe darse la acción educativa. 
A partir de estas líneas de trabajo puede plantearse la pertinencia de iniciar procesos de diseño y construcción de doble grado Pedagogía y Educación Social aún hoy inexistente. En primer lugar por la situación social y demanda de un perfil profesional para salvaguardar la equidad en el Estado de Bienestar. Y en segundo lugar por la fisonomía de ambas titulaciones.

Respecto a esto último, del total de grupos de asignaturas estudiados con mayor frecuencia de repetición en Pedagogía (82) y del total de grupos estudiados con el mismo criterio en Educación Social (59), podemos considerar que existe un $51,2 \%$ de asignaturas coincidentes. Además, también se observan asignaturas coincidentes con baja frecuencia de repetición en ambos grados, que no han sido recogidas por su baja representatividad grupal.

Juntos, como además ya se representara en el Libro Blanco de los títulos de Grado de Pedagogía y Educación Social, actúan en ámbitos y mediante perfiles profesionales altamente pertinentes en beneficio de la equidad social (Vila, 2005):

- Administración y Gestión Educativa (Gestor de centros educativos; Inspector y supervisor de la administración educativa; Evaluador de sistemas e instituciones educativas).

- Orientación e intervención psicopedagógica (Orientador personal, académico y profesional; Orientador familiar).

- Diseño, desarrollo y evaluación de procesos y medios educativos (Diseñador y evaluador de recursos curriculares, didácticos y tecnológicos; Diseñador y evaluador de procesos de enseñanza-aprendizaje; Formador pedagógico de la función docente).

- Formación y desarrollo en organizaciones (Consultor y gestor de formación en las organizaciones; Formador de formadores).

- Educación familiar y desarrollo comunitario (Educador de familia; Agente socioeducativo de desarrollo comunitario).

- Educación y mediación para la integración social (Educador de familia; Diseñador y evaluador de procesos de integración social).

- Educación del ocio, animación y gestión sociocultural (Educador en tiempo libre y ocio; Animador sociocultural; Gestor de programas y recursos socio culturales).

- Intervención socioeducativa en la infancia y juventud (Educador en instituciones de atención e inserción social; Mediador en procesos de acogida y adopción).

- Educación de personal adultas y mayores (Orientador socio-laboral; Educador de personas adultas y mayores).

- Atención socioeducativa a la diversidad (Pedagogo especialista en atención a la diversidad educativa; Dinamizador para la inserción sociolaboral).

Vistas las necesidades de la sociedad y la demanda de profesionales de este calado, la universidad puede plantear esta variante, esta evolución. La coincidencia en los ámbitos de intervención, asignaturas, y escenarios puede hacer factible este proceso de convergencia. Ello permitiría reforzar aún más de base teórica la vertiente educativo-social y por otro lado reforzar el cariz práctico, de los estudios de pedagogía, dotándoles de más flexibilidad. Lo atractivo de estos estudios radica en la fácil integralidad de los conocimientos por su coincidencia, lo que generaría una rentabilidad espacio temporal: profesionales formados más rápidamente en una vertiente más compleja y a la vez más directa para hacer frente a los retos de la sociedad. 


\section{REFERENCIAS BIBLIOGRÁFICAS}

Cáride, J. A. (2002). Construir la profesión: la Educación Social como proyecto ético y tarea cívica. Pedagogía Social. Revista Interuniversitaria, 9, 91-125.

Castillo Sánchez, M. y Gamboa Araya, R. (2012). Desafíos de la educación en la sociedad actual. Revista Electrónica Diálogos Educativos, 12(24),55-69.

De la Calle Velasco, M. J. (2004). El reto de ser profesor en el contexto de la convergencia europea. La formación pedagógica como necesidad. Revista Interuniversitaria de Formación del profesorado, 18(3), 251-258.

Fernández-García, T. (2012). El Estado de Bienestar frente a la crisis política, económica y social. Portularia, 12,3-12.

García Aretio, L., Ruiz Corbella, M., y García Blanco, M. (2011). Claves para la educación. Actores, agentes y escenarios en la sociedad actual. Madrid: Narcea UNED.

Houssaye, J. (2014). Formar en pedagogía: Sí, ¿ pero cómo?. Estudios pedagógicos, 9 (40), 275- 283.

Martínez García, R. (2018). La escuela en crisis: Una aproximación en clave socioeducativa. Revista Educación, Política y Sociedad, 3 (1), 31-49.

Ortega Esteban, E. (2005).Pedagogía social y pedagogía escolar: la educación social en la escuela. Revista de educación, (336), 111-127.

Pérez Serrano, G. (2005). Presentación. Revista de educación, 336, 7-18.

Puelles Benítez, M., y Torreblanca, J. I. (1995). Educación, desarrollo y equidad social. Revista Iberoamericana de Educación, 9, 165-189.

Puelles, M. (2007). ¿Pacto de Estado? La educación entre el consenso y el disenso. Revista de Educación, 344, 23-40.

Ramió, Matas, C. (2013). Balance del impacto de las crisis económica, política, social e institucional sobre las administraciones públicas en España 2011-2013: Diagnóstico y propuestas. GIGAPP Estudios Working Papers, (28),1-33.

Sánchez Alba, B., Gómez Jarabo, I., Sabán Vera, C., y Sáenz-Rico de Santiago, B. (2017). Sostenibilización del perfil profesional del educador social. Necesidades y demandas compartidas. Revista Iberoamericana de educación, (73), 109-130.

Senent Sánchez, J.M. (2005). Los estudios de Pedagogía en Europa en el contexto dela implantación del proceso de Bolonia y la situación de la Educación Comparada. Revista española de educación comparada, (11),95-133.

Úcar, X. (2011). Relaciones entre académicos y prácticos en el campo de la pedagogía social y de la educación social. En: M. Belando (coord.) II Jornada Monográfica «Pedagogía Social y Educación social: una mirada al futuro», (91-99). Madrid: Universidad Complutense de Madrid.

Universidad Abierta de Cataluña. Recuperado el 2/03/2018 http://www.uoc.edu/portal/es/Universidad Nacional de Educación a Distancia. Recuperado el 2/03/2018 http://portal.uned.es/portal/page?_pageid=93,1\&_dad=portal\&_ schema=PORTAL.

Universidad Complutense de Madrid. Recuperado el 2/03/2018 https://www.ucm.esUniversidad Politécnica de Valencia. Recuperado el 2/03/2018 https://www.upv.esUniversidad de Barcelona. Recuperado el 2/03/2018 http://www.ub.edu/ web/ub/ca/Universidad de Valencia. Recuperado el 2/03/2018 https://www.uv.es.

Universidad Internacional de La Rioja. Recuperado el 2/03/2018 https://www.unir.netUniversidad Rey Juan Carlos I. Recuperado el 2/03/2018 https://www.urjc.esUniversidad de Jaén. Recuperado el 8/03/2018 https://www.uja.es.

Universidad de Granada. Recuperado el 8/03/2018 https://www.ugr.es. Universidad Autónoma de Barcelona. Recuperado el 8/03/2018 http://www.uab.cat. Universidad de Málaga. Recuperado el 8/03/2018 https://www.uma.es.

Universidad Autónoma de Madrid. Recuperado el 8/03/2018 http://www.uam.es/UAM/Home.htm?language=es.

Universidad de Oviedo. Recuperado el 8/03/2018 http://www.uniovi.es. Universidad de Sevilla. Recuperado el 8/03/2018 http://www.us.es.

Universidad Pompeu Fabra. Recuperado el 8/03/2018 http://bcu.cat/upf-universitat-pompeu-fabra/.

Universidad Politécnica de Madrid. Recuperado el 2/03/2018 http://www.upm.es. Universidad de Salamanca. Recuperado el 2/03/2018 http://www.usal.es. Universidad de Alicante. Recuperado el 2/03/2018 https://www.ua.es. Universidad de Zaragoza. Recuperado el 2/03/2018 https://www.unizar.es.

Universidad de Santiago de Compostela. Recuperadoel 2/03/2018 http://www.usc.es/es/index.html.

Universidad de Almería. Recuperado el 8/03/2018 https://www.ual.es.

Universidad Europea de Madrid. Recuperado el 8/03/2018 https://madrid.universidadeuropea.es. Universidad Carlos III de Madrid. Recuperado el 2/03/2018 https://www.uc3m.es/Inicio. Universidad de La Laguna. Recuperado el 8/03/2018 https://www.ull.es.

Universidad Católica de Murcia. Recuperado el 8/03/2018 http://www.ucam.edu.Universidad Politécnica de Cataluña. Recuperado el 2/03/2018 https://www.upc.edu/ca. Universidad de Extremadura. Recuperado el 8/03/2018 https://www.unex.es. 
Universidad Internacional de Valencia. Recuperado el 8/03/2018 https://www.universidadviu.es. Universidad de Islas Baleares. Recuperado el 8/03/2018 http://www.uib.es/es/.

Universidad Jaume I. Recuperado el 8/03/2018 https://www.uji.es.

Universidad de las Palmas de Gran Canaria. Recuperado el 8/03/2018 https://www.ulpgc.es. Universidad de Girona. Recuperado el 8/03/2018 https://www.udg.edu/ca/.

Universidad Pública de Navarra. Recuperado el 8/03/2018 http://www.unavarra.es. Universidad de la Rioja. Recuperado el 12/03/2018 http://www.unirioja.es. Universidad Antonio de Nebrija. Recuperado el 12/03/2018 https://www.nebrija.com.

Universidad de Valladolid. Recuperado el 12/03/2018 http://www.uva.es/export/sites/uva/Universidad de Murcia. Recuperado el 12/03/2018 http://www.um.es

Universidad Francisco de Vitoria. Recuperado el 12/03/2018 http://www.ufv.esUniversidad de León. Recuperado el 15/03/2018 https://www.unileon.esUniversidad de Cádiz. Recuperado el 19/03/2018 http://www.uca.esUniversidad de Vigo. Recuperado el 19/03/2018 https://uvigo.gal

Universidad Ramón Llull. Recuperado el 19/03/2018 http://www.url.edu/esUniversidad Isabel I. Recuperado el 19/03/2018 https://www.ui1.es.

Universidad CEU San Pablo. Recuperado el 19/03/2018 https://www.uspceu.com/es/oferta-academica/index.aspx.

Universidad a Distancia de Madrid. Recuperado el 19/03/2018 https://www.udima.esUniversidad de Burgos. Recuperado el 19/03/2018 http://www.ubu.es.

Universidad Camilo José Cela. Recuperado el 19/03/2018 https://www.ucjc.eduUniversidad Pontificia de Salamanca. Recuperado el 19/03/2018 https://www.upsa.es

Universidad Pablo de Olavide. Recuperado el 19/03/2018 https://www.upo.es/portal/impe/web/portada.

Universidad Rovira y Virgil. Recuperado el 19/03/2018 http://www.urv.cat/es/Universidad del País Vasco. Recuperado el 19/03/2018 https://www.ehu.eus/es/.

Universidad Católica de Valencia San Vicente Mártir. Recuperado el 19/03/2018 https://www.ucv.es/oferta-academica/grados.

Universidad de Córdoba. Recuperado el 19/03/2018 http://www.uco.es. Universidad de La Coruña. Recuperado el 15/03/2018 https://www.udc.es. Universidad de Lleida. Recuperado el 15/03/2018. http://www.udl.es/ca/Universidad de Huelva. Recuperado el 15/03/2018 http://www.uhu.es/index.php.

Universidad de Castilla La Mancha. Recuperado el 15/03/2018 https://www.uclm.es. Universidad Central de Cataluña. Recuperado el 15/03/2018 https://www.uvic.cat/es.

Universidad de Deusto. Recuperado el 15/03/2018 https://www.deusto.es/cs/Satellite/deusto/es/universidad-deusto.

Villa, A. (coord.) (2005). Libro Blanco Titulo de Grado en Pedagogía y Educación Social. Madrid: ANECA (2 vols.).

Viñao, A. (2009). El Grado de Pedagogía y la formación del profesorado ante el Espacio Europeo de Educación Superior. Cuestiones pedagógicas, (19), 97-113. 